\title{
Precise Hsu's method for analyzing the stability of periodic solutions of multi-degrees-of-freedom systems with cubic nonlinearity
}

\author{
J.L. Huang ${ }^{\mathrm{a}}$, R.K.L. Su ${ }^{\mathrm{b}}$, S.H. Chen ${ }^{\mathrm{a}}$ \\ ${ }^{a}$ Department of Applied Mechanics and Engineering, Sun Yat-sen University, Guangzhou 510275, PR China \\ ${ }^{\mathrm{b}}$ Department of Civil Engineering, The University of Hong Kong, Pokfulam Road, Hong Kong, PR China
}

\begin{abstract}
This paper presents a new precise Hsu's method for investigating the stability regions of the periodic motions of an undamped two-degrees-of-freedom system with cubic nonlinearity. Firstly, the incremental harmonic balance (IHB) method is used to obtain the solution of nonlinear vibration differential equations. Hsu's method is then adopted for computing the transition matrix at the end of one period, and the precise time integration algorithm is adjusted to improve the computational precision. The stability regions of the system obtained from the precise Hsu's, Hsu's and improved numerical integration methods are compared and discussed.
\end{abstract}

Keywords: Incremental harmonic balance method, Hsu's method, Precise time integration algorithm, stability analysis.

\section{Introduction}

Many practical non-linear systems, such as rotorcraft and stability of a clamped-hinged beam, can be modeled by ordinary differential equations with periodic coefficients

$$
\dot{\mathbf{x}}=\mathbf{A}(t) \mathbf{x}, \mathbf{A}(t+T)=\mathbf{A}(t)
$$

where $\mathbf{x}$ is an $n$-dimensional vector, $\mathbf{A}$ is an $n \times n$ periodic matrix with period $T$, and $\dot{\mathbf{x}}=\mathrm{d} \mathbf{x} / \mathrm{d} t$.

Based on the Floquet-Liapunov theorem, the stability criteria of the system are related to the eigenvalues of the transition matrix. Two types of efficient numerical methods were developed for evaluating the transition matrix at the end of a periodic system. In the first type, Hsu [1-3] developed an efficient method for approximating the transition matrix during one period. To evaluate the transition matrix, this method divides the time interval of a period into a number of equal parts and considers the equations over each interval to be a set of equations with constant coefficients. Friedmann et al. [4] summarized this method and provided a clear and concise formulation. Cheung et al. [5] applied Hsu's method to treat the nonlinear vibration of strongly nonlinear multiple-degreesof-freedom systems with cubic nonlinearity based on the IHB method steady-state solutions. In the

*Corresponding author. Tel: +852 2859 2648; fax: +852 25595337.

E-mail address: klsu@hkucc.hku.hk 
second type, direct numerical integration is applied directly to solve the ordinary differential equations. Friedmann et al. [4] also improved the numerical integration scheme for evaluating the transition matrix based on the fourth-order Runge-Kutta scheme with Gill coefficients. Cai et al. [6] presented a new approach for computing the Floquet transition matrix based on the precise integration method.

In 1994, Zhong [7] and Zhong and Williams [8] proposed a precise time integration algorithm. Using this method, one can get accurate solutions by making use of only one time step over a large interval. This was developed, and successfully applied to structural dynamics and its related problems. Recently, Fung and Chen [9] improved the computational efficiency of the method by combining the Krylov subspace method and the Padé approximations for solving large-scale transient problems. To avoid the matrix inversion, Zhang et al. [10] used precise time integration with the Runge-Kutta method to solve nonlinear dynamical systems. Huang et al. [11] presented an improved symplectic precise integration method to increase the accuracy of the rotating rigid-flexible coupled system. Wang and Au [12] improved the accuracy of the precise time integration with the Gauss quadrature method. Tang [13] used precise time integration to compute the forced vibration of a beam with high precision. Chen et al. [14] applied the precise time method to solve linear two-point boundary value problems.

In this paper, we try to extend the precise time integration algorithm to deal with the stability of a clamped-hinged beam, which is a typical example of a system with cubic nonlinearity possessing internal resonance. The proposed approach is based on the incremental harmonic balance (IHB) method, Hsu's method, and the precise time integration algorithm (PTIA). The main objective of this study is to identify stability regions of the system with various combinations of excitation frequencies and amplitudes of the oscillations. In this approach, the IHB method is derived for a general nonlinear system to obtain the steady-state periodic solutions. Then, taking increments as perturbations of the solutions for the ordinary differential equations, one can obtain a set of linear variational equations with periodic coefficients that can be used to determine the stability characteristics. Hsu's method, which is compounded with the precise time integration algorithm, is used to determine the stability of the system based on the Floquet theorem. This new approach is named the precise Hsu's method. The stability regions of the system are evaluated using the precise Hsu's method, Hsu's method and improved numerical integration method which is based upon the fourth order Runge-Kutta scheme with Gill coefficients [4]. The accuracy of results obtained from various approaches are compared and discussed.

\section{Incremental Harmonic Balance Method}

Consider a nonlinear system in the general form given by the equation:

$$
\mathbf{f}(\ddot{\mathbf{q}}, \dot{\mathbf{q}}, \mathbf{q}, \mathbf{F}, \omega, \tau)=\mathbf{0}
$$

where $\mathbf{q}$ is an $N$-dimensional displacement vector, e.g., $\mathbf{q}=\left[q_{1}, q_{2}, \ldots, q_{N}\right]^{T}, \mathbf{F}=\left[F_{1}, F_{2}, \ldots, F_{N}\right]^{T}$ is an $N$-dimensional load vector, $\dot{\mathbf{q}}=\mathrm{d} \mathbf{q} / \mathrm{d} \tau, \mathbf{f}$ is a set of second order nonlinear ordinary differential equations, and the time variable $\tau$ is defined as $\tau=\omega t$.

Among the solution methods of ordinary differential equations, the IHB method is relatively efficient and reliable for treating the vibrations of strongly nonlinear systems to any desired accuracy. The method has been developed and successfully applied to analyze periodic and almost periodic nonlinear structural vibrations and their related problems [5, 15, 16, 17, 18, 19]. 
The first step of the IHB method is the incremental procedure. Let $\mathbf{q}_{0}, \omega_{0}$ and $\mathbf{F}_{0}$ denoted an initial guess state of vibration, then a neighboring state of motion can be expressed by adding the corresponding increments as:

$$
q_{j}=q_{j 0}+\Delta q_{j}, j=1,2, \ldots, N, \quad \omega=\omega_{0}+\Delta \omega, \quad F_{i}=F_{i 0}+\Delta F_{i}, i=1,2, \ldots, N
$$

Substituting Equation (3) into Equation (2) and using a Taylor expansion, and neglecting small terms of the higher order terms in $\Delta \mathbf{q}, \Delta \omega$ and $\Delta \mathbf{F}$, one obtains the linearized incremental equation in matrix form:

$$
(\partial \mathbf{f} / \partial \ddot{\mathbf{q}})_{0} \Delta \ddot{\mathbf{q}}+(\partial \mathbf{f} / \partial \dot{\mathbf{q}})_{0} \Delta \dot{\mathbf{q}}+(\partial \mathbf{f} / \partial \mathbf{q})_{0} \Delta \mathbf{q}=\overline{\mathbf{R}}\left(\mathbf{q}_{0}, \mathbf{F}_{0}, \omega_{0}\right)-(\partial \mathbf{f} / \partial \mathbf{F})_{0} \Delta \mathbf{F}-(\partial \mathbf{f} / \partial \omega)_{0} \Delta \omega
$$

where $\overline{\mathbf{R}}\left(\mathbf{q}_{0}, \mathbf{F}_{0}, \omega_{0}\right)=-\mathbf{f}\left(\mathbf{q}_{0}, \mathbf{F}_{0}, \omega_{0}\right)$ is a residual/corrective vector that goes to zero when the numerical solution is exact. In this sense, the residual term can be used to control the accuracy in the computation.

The second step of the IHB method is the Galerkin procedure (i.e., the harmonics balance procedure), we assume a steady-state solution of an $N$-dimensional nonlinear system and its increment expanded in a finite Fourier series as follow:

$$
\begin{gathered}
q_{j 0}=\sum_{k=1}^{n_{c}} a_{j k} \cos (k-1) \tau+\sum_{k=1}^{n_{s}} b_{j k} \sin k \tau=\mathbf{C A}_{j} \\
\Delta q_{j 0}=\sum_{k=1}^{n_{c}} \Delta a_{j k} \cos (k-1) \tau+\sum_{k=1}^{n_{s}} \Delta b_{j k} \sin k \tau=\mathbf{C} \Delta \mathbf{A}_{j}
\end{gathered}
$$

where $n_{c}, n_{s}$ are positive integers, represent the numbers of cosine and sine harmonic terms, respectively. And the vectors $\mathbf{C}, \mathbf{A}_{j}, \Delta \mathbf{A}_{j}$ are given by

$$
\begin{gathered}
\mathbf{C}=\left[1, \cos \tau, \cos 2 \tau \cdots, \cos \left(n_{c}-1\right) \tau, \sin \tau, \sin 2 \tau, \cdots, \sin n_{s} \tau\right], \\
\mathbf{A}_{j}=\left[a_{j 1}, a_{j 2}, \ldots, a_{j n_{c}}, b_{j 1}, b_{j 2}, \ldots, b_{j n_{s}}\right]^{T}, \\
\Delta \mathbf{A}_{j}=\left[\Delta a_{j 1}, \Delta a_{j 2}, \ldots, \Delta a_{j n_{c}}, \Delta b_{j 1}, \Delta b_{j 2}, \ldots, \Delta b_{j n_{s}}\right]^{T}
\end{gathered}
$$

Hence, the vectors of unknown and their increments can be expressed by the Fourier coefficients vector $\mathbf{A}$ and its increment $\Delta \mathbf{A}$ as follows:

$$
\mathbf{q}_{0}=\mathbf{S A}, \quad \Delta \mathbf{q}_{0}=\mathbf{S} \Delta \mathbf{A}
$$

where

$$
\mathbf{S}=\operatorname{diag}(\mathbf{C}, \mathbf{C}, \cdots, \mathbf{C}), . \mathbf{A}=\left[\mathbf{A}_{1}, \mathbf{A}_{2}, \cdots, \mathbf{A}_{N}\right]^{T}, \Delta \mathbf{A}=\left[\Delta \mathbf{A}_{1}, \Delta \mathbf{A}_{2}, \cdots, \Delta \mathbf{A}_{N}\right]^{T}
$$

Substituting Equations (7) and (8) into Equation (4) and using the Galerkin procedure in order to harmonics balance yields

$$
\begin{aligned}
\int_{0}^{2 \pi} \delta(\Delta \mathbf{q})^{T}[ & \left.(\partial \mathbf{f} / \partial \ddot{\mathbf{q}})_{0} \Delta \ddot{\mathbf{q}}+(\partial \mathbf{f} / \partial \dot{\mathbf{q}})_{0} \Delta \dot{\mathbf{q}}+(\partial \mathbf{f} / \partial \mathbf{q})_{0} \Delta \mathbf{q}\right] \mathrm{d} \tau \\
& =\int_{0}^{2 \pi} \delta(\Delta \mathbf{q})^{T}\left(\overline{\mathbf{R}}\left(\mathbf{q}_{0}, \mathbf{F}_{0}, \omega_{0}\right)-(\partial \mathbf{f} / \partial \mathbf{F})_{0} \Delta \mathbf{F}-(\partial \mathbf{f} / \partial \omega)_{0} \Delta \omega\right) \mathrm{d} \tau
\end{aligned}
$$

One can easily obtain a set of linear equations in terms of $\Delta \mathbf{A}, \Delta \omega$ and $\Delta \mathbf{F}$ as follow:

$$
\mathbf{K} \Delta \mathbf{A}=\mathbf{R}-\mathbf{P} \Delta \mathbf{F}-\mathbf{C} \Delta \omega
$$

where

$$
\mathbf{K}=\int_{0}^{2 \pi} \mathbf{S}^{T}(\partial \mathbf{f} / \partial \ddot{\mathbf{q}})_{0} \ddot{\mathbf{S}} \mathrm{d} \tau+\int_{0}^{2 \pi} \mathbf{S}^{T}(\partial \mathbf{f} / \partial \dot{\mathbf{q}})_{0} \dot{\mathbf{S}} \mathrm{d} \tau+\int_{0}^{2 \pi} \mathbf{S}^{T}(\partial \mathbf{f} / \partial \mathbf{q})_{0} \mathbf{S} \mathrm{d} \tau
$$




$$
\mathbf{R}=\int_{0}^{2 \pi} \mathbf{S}^{T} \overline{\mathbf{R}}\left(\mathbf{q}_{0}, \mathbf{F}_{0}, \omega_{0}\right) \mathrm{d} \tau, \mathbf{P}=\int_{0}^{2 \pi} \mathbf{S}^{T}(\partial \mathbf{f} / \partial \mathbf{F})_{0} \mathrm{~d} \tau, \mathbf{C}=\int_{0}^{2 \pi} \mathbf{S}^{T}(\partial \mathbf{f} / \partial \omega)_{0} \mathrm{~d} \tau
$$

It is worth mentioning that in Equation (10) the number of incremental unknown is greater than the number of equations available due to the existence of $\Delta \mathbf{F}$ and $\Delta \omega$. However, since one is primarily interested in the frequency-response curves of the system for a constant force level, $\mathbf{F}$ is fixed as a parameter vector, which implies $\Delta \mathbf{F}=\mathbf{0}$. Hence Equation (10) is reduced to

$$
\mathbf{K} \Delta \mathbf{A}=\mathbf{R}-\mathbf{C} \Delta \omega
$$

The solution process begins with a guessed solution. The nonlinear frequency-amplitude response curve is then solved point-by-point by incrementing the frequency $\omega$ or incrementing component of the coefficient vector A . The Newton-Raphson iterative method can be employed. Hence, the solution of the differential equation can be found.

\section{The precise Hsu's method and the stability of the steady-state solutions}

\subsection{Solution stability analysis}

When the steady state solution of a nonlinear system is obtained by the IHB method, the stability of the given solution can be investigated by adding a small perturbation $\Delta \mathbf{q}$ on $\mathbf{q}_{0}$, i.e. by letting

$$
\mathbf{q}=\mathbf{q}_{0}+\Delta \mathbf{q}
$$

Substituting Equation (12) into Equation (2), linearizing the resulting equation in terms of $\Delta \mathbf{q}$ and noting that $\mathbf{q}_{0}$ satisfies Equation (2), one can obtain the linearized equation for $\Delta \mathbf{q}$ :

$$
(\partial \mathbf{f} / \partial \ddot{\mathbf{q}})_{0} \Delta \ddot{\mathbf{q}}+(\partial \mathbf{f} / \partial \dot{\mathbf{q}})_{0} \Delta \dot{\mathbf{q}}+(\partial \mathbf{f} / \partial \mathbf{q})_{0} \Delta \mathbf{q}=\mathbf{0}
$$

Equation (13) is the perturbed equation: i.e., perturbed from the known solution $\mathbf{q}_{0}$. The stability of the steady-state solutions corresponds to the stability of the solutions of Equation (13), which is a set of linear ordinary differential equations. The stability characteristics can be studied by multi-variable Floquet theory. Let

$$
\mathbf{X}=[\Delta \mathbf{q}, \Delta \dot{\mathbf{q}}]^{T}
$$

Equation (13) can be rewritten in the state variable form as:

$$
\text { where } \mathbf{Q}=\left[\begin{array}{cc}
\mathbf{0} & \dot{\mathbf{X}}=\mathbf{Q}(\tau) \mathbf{X} \\
-(\partial \mathbf{f} / \partial \ddot{\mathbf{q}})_{0}^{-1}(\partial \mathbf{f} / \partial \mathbf{q})_{0} & -(\partial \mathbf{f} / \partial \ddot{\mathbf{q}})_{0}^{-1}(\partial \mathbf{f} / \partial \dot{\mathbf{q}})_{0}
\end{array}\right]
$$

Since each component of $\mathbf{q}_{0}$ is a periodic function $\tau$ with a period $T=2 \pi$, each element of $\mathbf{Q}$ is also a periodic function with the same period $T$.

For Equation (15), there exists a fundamental set of solutions

$$
\mathbf{y}_{k}=\left[y_{1 k}, y_{2 k}, \cdots, y_{M k}\right]^{T}, k=1,2, \cdots, M
$$

where $M=2 N$. This fundamental set can be expressed in a matrix called a fundamental matrix solution:

$$
\mathbf{Y}=\left[\begin{array}{cccc}
y_{11} & y_{12} & \cdots & y_{1 M} \\
y_{21} & y_{22} & \cdots & y_{2 M} \\
\vdots & \vdots & \cdots & \vdots \\
y_{M 1} & y_{M 2} & \cdots & y_{M M}
\end{array}\right]
$$


Y satisfies the matrix equation

$$
\dot{\mathbf{Y}}=\mathbf{Q}(\tau) \mathbf{Y}
$$

where $\mathbf{Q}(\tau+T)=\mathbf{Q}(\tau)$ is the periodic matrix, and $\mathbf{Y}(\tau+T)$ is a fundamental matrix solution, so it can be expressed by

$$
\mathbf{Y}(\tau+T)=\mathbf{P Y}(\tau)
$$

where $\mathbf{P}$ is a non-singular constant matrix called the transition matrix.

\subsection{Hsu's method}

The Floquet theory states that the stability criteria for the system are related to the eigenvalues of the matrix $\mathbf{P}$ or the real of part of the characteristic exponents. The solution of Equation (15) approaches zero as $\tau \rightarrow \infty$ if all of the moduli of the eigenvalues $\lambda_{i}$ of $\mathbf{P}$ are less than 1 , and otherwise the motion is unbounded and the solution is unstable. If $\lambda_{i}=1, \mathbf{X}$ is periodic with period $T$, while if $\lambda_{i}=-1, \mathbf{X}$ is periodic with period $2 T$.

Among the various methods for approximating the transition matrix $\mathbf{P}$, Friedmann et al. [4] reported that the most efficient procedure was that developed by Hsu [1-3]. The method consists of evaluating the transition matrix, by dividing a period into a number of equal parts and considering the equations over each interval to be a set of equations with constant coefficients. Suppose each period $T=2 \pi$ is divided into $K$ intervals denoted by $\tau_{k}, k=0,1,2, \ldots, K$ with $0<\tau_{0}<\tau_{1}<\ldots<\tau_{K}=T$ and the size of the $k$ th interval is denoted by:

$$
\Delta_{k}=\tau_{k}-\tau_{k-1}
$$

In the $k$ th interval, the periodic coefficient matrix $\mathbf{Q}(\tau)$ is replaced by its average value, i.e. by a constant matrix $\mathbf{Q}_{k}$ defined by:

$$
\mathbf{Q}_{k}=\frac{1}{\Delta_{k}} \int_{\tau_{k-1}}^{\tau_{k}} \mathbf{Q}(\zeta) \mathrm{d} \zeta
$$

For the $i$ th interval, the approximate transition matrix can be expressed as

$$
\mathbf{Y}_{i}=\exp \left(\mathbf{Q}_{i} \cdot \Delta_{i}\right)
$$

Finally, the transition matrix $\mathbf{P}$ is given in the form

$$
\mathbf{P}=\prod_{i=1}^{K} \exp \left(\mathbf{Q}_{i} \cdot \Delta_{i}\right)=\prod_{i=1}^{K} \mathbf{Y}_{i}
$$

The multiplication ordering sequence is such that the $k$ th factor must be placed in front of the (k-1)th factor in the Equation (23) product sign.

In Reference [4], the matrix exponential is evaluated by an $N_{j}$ th order truncated Taylor series

$$
\exp \left(\mathbf{Q}_{i} \cdot \Delta_{i}\right)=\mathbf{I}+\sum_{j=1}^{\infty} \frac{\left(\Delta_{i} \mathbf{Q}_{i}\right)^{j}}{j !} \square \mathbf{I}+\sum_{j=1}^{N_{j}} \frac{\left(\Delta_{i} \mathbf{Q}_{i}\right)^{j}}{j !}
$$

and the transition matrix $\mathbf{P}$ can be rewritten as

$$
\mathbf{P}=\mathbf{Y}(T)=\prod_{i=1}^{K}\left[\mathbf{I}+\sum_{j=1}^{N_{j}} \frac{\left(\Delta_{i} \mathbf{Q}_{i}\right)^{j}}{j !}\right]
$$

where $\mathbf{I}$ is a unit matrix.

The above representations for the matrix exponential and the matrix multiplication are conceptually simple and basis for an algorithm. As shown by Moler and Van Loan [20], the method employing the 
Taylor series expansion may be unsatisfactory due to round-off error difficulties. How to improve the accuracy in evaluating the periodic fundamental matrix $\exp \left(\mathbf{Q}_{i} \cdot \Delta_{i}\right)$ plays an important role in stability analysis.

\subsection{The precise Hsu's method}

To improve Hsu's method, two procedure steps which differ from the original method are included. The first one is to compute the matrix exponential $\exp \left(\mathbf{Q}_{i} \cdot \Delta_{i}\right)$ by the precise time integration algorithm given in References [7,8], instead of directly evaluated by the Taylor series expansion, such that the matrix exponential $\exp \left(\mathbf{Q}_{i} \cdot \Delta_{i}\right)$ of Equation (22) can be rewritten as:

$$
\mathbf{Y}_{i}=\exp \left(\mathbf{Q}_{i} \cdot \Delta_{i}\right)=\left[\exp \left(\mathbf{Q}_{i} \cdot \Delta_{i} / m\right)\right]^{m}
$$

where $m=2^{N}$.

It is noted that the matrix exponential $\mathbf{Y}_{i}$ in Equation (26) can be evaluated also through the knowledge of the eigenproperties of the matrix $\mathbf{Q}_{i}$.However, when the matrix $\mathbf{Q}_{i} \Delta_{i}$ has large elements, the method based on eigenvalue decompositions of the matrix $\mathbf{Q}_{i}$ may be adversely affected by round-off error, therefore, the traditional technique will fail in solution stability analysis. Letting $\Delta_{t}=\Delta_{i} / m, \Delta_{i}=2 \pi / K$ and then using the Taylor series expansion, we obtain:

$$
\exp \left(\mathbf{Q}_{i} \cdot \Delta_{t}\right)=\mathbf{I}+\mathbf{Q}_{i} \cdot \Delta_{t}+\frac{\left(\mathbf{Q}_{i} \cdot \Delta_{t}\right)^{2}}{2 !}+\cdots+\frac{\left(\mathbf{Q}_{i} \cdot \Delta_{t}\right)^{n}}{n !}+\cdots
$$

For very small time intervals, $\Delta_{t} \rightarrow 0$, the series in Equation (27) converges rapidly. Hence for the interval, the value of the matrix exponential can be approximated with high precision by a finite number of terms, as follow:

$$
\exp \left(\mathbf{Q}_{i} \cdot \Delta_{t}\right) \approx \mathbf{I}+\sum_{j=1}^{n} \frac{\left(\Delta_{t} \cdot \mathbf{Q}_{i}\right)^{j}}{j !}=\mathbf{I}+\mathbf{T}_{a}
$$

Note the norm of matrix $\mathbf{T}_{a}$ compared to 1 is very small (about $2 \times 10^{-7}$ in the next section numerical examples) and the orders of the elements magnitude of the matrix $\mathbf{T}_{a}$ are from $10^{-15}$ to $10^{-6}$ in the numerical examples. The subscript $a$ in $\mathbf{T}_{a}$ means approximation. Thus Equation (26) becomes

$$
\mathbf{Y}_{i}=\exp \left(\mathbf{Q}_{i} \cdot \Delta_{i}\right)=\left[\mathbf{I}+\mathbf{T}_{a}\right]^{2^{N}}=\left[\mathbf{I}+\mathbf{T}_{a}\right]^{2^{N-1}} \times\left[\mathbf{I}+\mathbf{T}_{a}\right]^{2^{N-1}}=\left[\mathbf{I}+2 \times \mathbf{T}_{a}+\mathbf{T}_{a} \times \mathbf{T}_{a}\right]^{2^{N-1}}
$$

This is also known as the scaling and squaring technique.

In the computation, we only stored the matrix $\mathbf{T}_{a}$ in the memory as an incremental part rather than the matrix $\mathbf{I}+\mathbf{T}_{a}$. Because matrix $\mathbf{T}_{a}$ is very small, if it is added to the unit matrix $\mathbf{I}, \mathbf{T}_{a}$ will become an appended part and its precision will be seriously dropped in round-off operations in computer arithmetic [21]. Therefore, Equation (29) becomes 


$$
\begin{aligned}
\mathbf{Y}_{i} & =\left(\mathbf{I}+\mathbf{T}_{a}^{i, 0}\right)^{2^{N}} \\
& =\left(\mathbf{I}+2 \times \mathbf{T}_{a}^{i, 0}+\mathbf{T}_{a}^{i, 0} \times \mathbf{T}_{a}^{i, 0}\right)^{2^{N-1}} \\
& =\left(\mathbf{I}+\mathbf{T}_{a}^{i, 1}\right)^{2^{N-1}} \\
& =\left(\mathbf{I}+2 \times \mathbf{T}_{a}^{i, 1}+\mathbf{T}_{a}^{i, 1} \times \mathbf{T}_{a}^{i, 1}\right)^{2^{N-2}}
\end{aligned}
$$

where the matrix $\mathbf{T}_{a}^{i, 0}$ is the initial matrix in the iterative computation, i.e. is equal to $\mathbf{T}_{a}$. Then, the following instruction can be executed

$$
\text { for }(l=0 ; l<N ; l++) \quad \mathbf{T}_{a}^{i, l+1}=2 \times \mathbf{T}_{a}^{i, l}+\mathbf{T}_{a}^{i, l} \times \mathbf{T}_{a}^{i, l}
$$

where the symbol $l++$ represents the increment operator by one the value of $l$.

Eventually, the matrix $\mathbf{Y}_{i}$ can be obtained from:

$$
\exp \left(\mathbf{Q}_{i} \cdot \Delta_{i}\right)=\mathbf{Y}_{i}=\mathbf{I}+\mathbf{T}_{a}^{i, N}
$$

Equations (28), (31) and (32) can be used to avoid the loss of significant digits in the matrix $\mathbf{Y}_{i}$ in the $i$ th interval. It is noted that the accuracy of the matrix $\mathbf{Y}_{i}$ depends on the size of time $\Delta_{t}=\Delta_{i} / \mathrm{m}$. Zhong [21] suggested the computer double precision, which has 16 decimal digits today, could be fully exploited as the number of the Taylor expansion terms $n=4$ in Equation (28) and the dimension of the time intervals $\Delta_{t}=\Delta_{i} / m, m=2^{20}=1048576$ in Equation (26). Hence the precise time integration algorithm has higher accuracy and there is no convergence error involved in the final results.

The second step is to compute the matrix multiplication, such that Equation (23) becomes

$$
\begin{aligned}
\mathbf{P} & =\prod_{i=1}^{K} \exp \left(\mathbf{Q}_{i} \cdot \Delta_{i}\right)=\prod_{i=1}^{K} \mathbf{Y}_{i}=\left(\mathbf{I}+\mathbf{T}_{a}^{K, N}\right)\left(\mathbf{I}+\mathbf{T}_{a}^{K-1, N}\right) \cdots\left(\mathbf{I}+\mathbf{T}_{a}^{1, N}\right) \\
& =\left(\mathbf{I}+\mathbf{T}_{a}^{K, N}+\mathbf{T}_{a}^{K-1, N}+\mathbf{T}_{a}^{K, N} \times \mathbf{T}_{a}^{K-1, N}\right)\left(\mathbf{I}+\mathbf{T}_{a}^{K-2, N}\right) \cdots\left(\mathbf{I}+\mathbf{T}_{a}^{1, N}\right)
\end{aligned}
$$

where $\mathbf{T}_{a}^{1, N}$ is $\mathbf{Y}_{i}-\mathbf{I}$.

In the computation, we also stored $\mathbf{T}_{a}^{i, N}+\mathbf{T}_{a}^{i-1, N}+\mathbf{T}_{a}^{i, N} \times \mathbf{T}_{a}^{i-1, N}$ into $\mathbf{T}_{a}^{i-1, N}$ as an incremental part and the following instruction can be executed

$$
\text { for }(i=K ; i>1 ; i--) \quad \mathbf{T}_{a}^{i-1, N}=\mathbf{T}_{a}^{i, N}+\mathbf{T}_{a}^{i-1, N}+\mathbf{T}_{a}^{i, N} \times \mathbf{T}_{a}^{i-1, N}
$$

where the symbol $i--$ represents the decrement value operator by one the value of $i$.

Finally, the transition matrix $\mathbf{P}$ can be obtained from:

$$
\mathbf{P}=\prod_{i=1}^{K} \exp \left(\mathbf{Q}_{i} \cdot \Delta_{i}\right)=\prod_{i=1}^{K} \mathbf{Y}_{i}=\mathbf{I}+\mathbf{T}_{a}^{1, N}
$$

where it is mentioned here that the matrix $\mathbf{T}_{a}^{1, N}$ in Equation (35), which is the matrix after compute iterations by instruction Equation (34), is different from the matrix $\mathbf{T}_{a}^{1, N}$ in Equation (33).

In general speaking, Hsu's method and the improved integration method in Reference [4] combined with multivariable Floquet theorem are efficient numerical methods available for dealing with the stability of periodic systems, however, when the moduli of the eigenvalues $\lambda_{i}$ of $\mathbf{P}$ are equal to 1.0 those methods are not suitable due to $\lambda_{i}>1.0$ at times with round-off errors. In the present procedure, the essential ideas are to subdivide the time interval $\Delta_{i}$ into a more subtle one $\Delta_{t}$ with the $2^{N}$ algorithm in Equation (26)and keep the small matrix $\mathbf{T}$ in the memory rather than the matrix $\mathbf{I}+\mathbf{T}$ as 
the incremental part in Equations (30) and (33) to minimize the round-off error. Therefore, the precise Hsu's method is more accurate than the other two methods in stability analysis of periodic systems.

\section{Numerical examples}

To examine the accuracy of the precise Hsu's method, we calculated a numerical example that involves forced vibrations of an undamped clamped-hinged beam. Using a two-mode shape approximation under a harmonic excitation, the governing dynamic equations of the problem can be expressed as follows:

$$
\begin{aligned}
& \frac{\mathrm{d}^{2} q_{1}}{\mathrm{~d} t^{2}}+\omega_{1}^{2} q_{1}+\alpha_{11} q_{1}^{3}+\alpha_{12} q_{1}^{2} q_{2}+\alpha_{13} q_{1} q_{2}^{2}+\alpha_{14} q_{2}^{3}=f_{1} \cos \Omega t \\
& \frac{\mathrm{d}^{2} q_{2}}{\mathrm{~d} t^{2}}+\omega_{2}^{2} q_{2}+\alpha_{21} q_{2}^{3}+\alpha_{22} q_{2}^{2} q_{1}+\alpha_{23} q_{2} q_{1}^{2}+\alpha_{24} q_{1}^{3}=f_{2} \cos \Omega t
\end{aligned}
$$

where $q_{1}$ and $q_{2}$ are normal mode coordinates, $\omega_{1}$ and $\omega_{2}$ are the first and second linear frequencies, respectively, $f_{1}$ and $f_{2}$ are forcing coefficients and $\Omega$ is the exciting frequency. The dimensionless quantities can be chosen as follows [16]:

$$
\begin{gathered}
\omega_{1}=1.0, \omega_{2}=3.2406, \alpha_{11}=0.278769, \alpha_{12}=-0.311074, \alpha_{13}=1.11585, \alpha_{14}=-0.386361, \\
\alpha_{21}=3.87030, \alpha_{22}=3 \alpha_{14}, \alpha_{23}=\alpha_{13}, \alpha_{24}=\frac{1}{3} \alpha_{12} .
\end{gathered}
$$

As Equations (36) and (37) should not contain quadratic nonlinear and damping terms, the solutions $q_{1}$ and $q_{2}$ can be expressed as follow:

$$
q_{j}=\sum_{k=1}^{n_{c}} a_{j(2 k-1)} \cos (2 k-1) \tau \quad j=1,2
$$

where $\tau=\omega_{1} t$. In order to obtain an accurate stability at a reasonable central processing unit (CPU) time, we choose the appropriate numbers of harmonic terms $n_{c}=8$ in Equation (38).

\subsection{Stability of fundamental resonance at $\Omega$ near $\omega_{1}$}

Figure 1 shows the response curves for the fundamental resonance with the IHB method when the forcing frequency $\Omega$ is near the first natural frequency $\omega_{1}$, and we use $f_{1}=0.03$ and $f_{2}=0$ in Equations (36) and (37). In addition, the second natural frequency of the systems is nearly three times the first natural frequency, i.e. $\omega_{2} \approx 3 \omega_{1}$, so internal resonance will occur.

Figure 1 shows the frequency response $\Omega-a_{11}$ and $\Omega-a_{23}$ curves, where $a_{11}$ and $a_{23}$ are defined in Equation (38) and are the amplitudes of the first and the third harmonic terms in the two modes $q_{1}$ and $q_{2}$, respectively. Both $a_{11}$ and $a_{23}$ possess three solutions, $a_{11}^{(1)}$ and $a_{23}^{(1)}, a_{11}^{(2)}$ and $a_{23}^{(2)}, a_{11}^{(3)}$ and $a_{23}^{(3)}$. Some of those solutions in difference phase, representing the "in-phase” and "out-of-phase” responses. The internal resonances, which provide the mechanism for transferring energy between the two modes, exist in both "in-phase" and "out-of-phase" planes. However, many portions of these curves correspond to unstable solutions, and hence cannot be realized in practice.

In this section, we use the precise Hsu's method to analyze the stability of the steady-state solution, compared with the improved numerical integration method based on the fourth order Runge-Kutta scheme with Gill coefficients and Hsu's method [4]. The stability of the steady-state solution is also 
shown in Figure 1, where the dashed and solid lines represent the unstable and stable solutions, respectively, determined by all three methods, and the thick lines represent the stability-disputed solutions regions (the three frequency ranges are $1.121<\Omega<1.241,1.156<\Omega<1.209$ and $1.083<\Omega<1.123$ in periodic solutions $a^{(1)}, a^{(2)}$ and $a^{(3)}$,respectively) which are stable determined by the precise Hsu's method, while are unstable determined by Hsu's method and improved numerical integration method up to Floquet theory with each period $T$ divided into the same $K$ intervals from 400 to 2000 . We should mention, for the interval $K<400$, that the convergence of the transition matrix $\mathbf{P}$ is not good for this particular problem using the three methods.

Now the problem is to check the stability (stable or unstable) in the stability-disputed regions. To analyze the stability in these regions, we choose three points of which the corresponding frequencies are $\Omega=1.2070, \Omega=1.1819$, and $\Omega=1.1165$ in periodic solutions $a^{(1)}, a^{(2)}$ and $a^{(3)}$. The character of the waveforms of the clamped-hinged beam is illustrated in Figure 2, where the solid line represents the response of mode $q_{1}$ and the dashed line represents the response of mode $q_{2}$. It is clear from the waveforms of $q_{1}$ and $q_{2}$ in Figure 2 that the solutions of the three points are stable.

It is worth noting that the waveforms $q_{1}$ and $q_{2}$ of the clamped-hinged beam oscillations in Figure 2 are periodic motions, with the periods $T$ and $T / 3\left(T=2 \pi / \Omega, \Omega \approx \omega_{1}\right)$, and hence all four moduli of the eigenvalues of Equation (19), which are induced by Equations (36) and (37), should be $\lambda_{i}=1.0(i=1,2,3,4)$. The moduli of the eigenvalues of the three points $(\Omega=1.2070, \Omega=1.1819$, and $\Omega=1.1165$ in periodic solutions $a^{(1)}, a^{(2)}$ and $a^{(3)}$, respectively) by the three methods are presented in the last three columns of Table I. It can be seen that the discrepancies of the results between the improved numerical integration and Hsu's method are quite small. The values of $\lambda_{1,2}$ are greater than 1.0, and the values of $\lambda_{3,4}$ are less than 1.0 with $K$ from 400 to 800 , so the solutions of the three points are unstable by using the two methods. In reality, the solutions are stable at the three points with $\lambda_{i}=1.0(i=1,2,3,4)$ in Figure 2 , which demonstrates that stability analyzed by the two latter methods is incorrect, but that the precise Hsu's method gives fairly good results.

We show in Table I, the values of $\lambda_{i}$ of the improved numerical integration and Hsu's method tend to be accurate for increasing $K$. However, for large values of $K$, such as at $K=5000$ in Table I, computational error appeared to be due to the very small value of the interval $\Delta_{k}$ used in the denominator of Equation (21). Hence, stability assessment for nonlinear vibration solutions would be wrong. This occurs not only in the improved numerical integration and Hsu's method, but also in the precise Hsu's method. We note that it is important to select the appropriate number of integral intervals $K$. If $K$ is a small value, the periodic coefficient matrix $\mathbf{Q}(\tau)$ in Equation (21) becomes too large to conduct stability analyses. However, if $K$ is very large, computational error appears. In this example and the follow sections, the reasonable region of $K$ is found to be from 400 to 2000 .

\subsection{Stability of fundamental resonance at $\Omega$ near $\omega_{2}$}

Figure 3 shows the $\Omega-a_{23}$ response curves for the fundamental resonance with the IHB method when $\Omega \approx \omega_{2}$, and $f_{1}=0, f_{2}=0.05$ are taken in Equations (36) and (37). This response is similar to the ordinary forced vibration in a single-degree-of-freedom Duffing system. In Figure 3, we note that stability analyzed in this response curve by the precise Hsu's method is similar to the other two methods. At the stable points of the response curve, the moduli of the eigenvalues are 
$\lambda_{i}=1.0(i=1,2,3,4)$ using the precise Hsu's method, whereas these moduli $\lambda_{i}$ are less than 1.0 , but tend to 1.0 using the other two methods with an increasing number of integral intervals $K$ from 400 to 2000.

\subsection{Stability of subharmonic resonance at $\Omega$ near $\omega_{2}$}

When the force frequency $\Omega$ is near the second natural frequency $\omega_{2}$, i.e. $\Omega \approx \omega_{2}$, and $f_{1}=0$, $f_{2}=0.05$ are taken in Equations (36) and (37), the other response of the system is a subharmonic resonance, in which the major response curves $\Omega-a_{11}$ and $\Omega-a_{23}$ of the modes $q_{1}$ and $q_{2}$ have two solutions, when using the IHB method, as shown in Figure 4. Note that the stabilities of many portions of these curves, determined by the three stability methods, are consistent besides the two stability-disputed regions, which are stable solutions using the precise Hsu's method, while unstable using the other two methods with the same integral intervals $K$ (from 400 to 2000). The first stability-disputed region ( $\Omega / 3 \omega_{1}$ from 1.1186 to 1.1265 ) exists in the top amplitude of the response curve $\Omega-a_{11}^{(1)}$, in which the internal resonance begins to happen. For the second periodic solution, as can be seen in Figure 4 (a) and (b), the stability-disputed region exists "out-of-phase" of the response curve $\Omega-a_{11}^{(2)}$ and "in phase" of the response curve $\Omega-a_{23}^{(2)}$ with $1.158 \leq \Omega / 3 \omega_{1} \leq 1.223$. In order to determine the real stability of the stability-disputed regions, we choose point $\Omega=3.356$ and point $\Omega=3.657$ from the two regions, respectively. Figure 5 shows that the waveforms $q_{1}$ and $q_{2}$ of the clamped-hinged beam oscillations are periodic motions at the two points, with periods $T$ and $T / 3$ ( $T=2 \pi / \Omega, \Omega \approx \omega_{2}$ ), respectively. It is clear from the waveforms $q_{1}$ and $q_{2}$ at the two points that the solutions of the stability-disputed regions are indeed stable. Therefore, the results reveal that the precise Hsu's method can correctly determine the stability of the periodic solutions of the subhamonic resonance.

\section{Conclusions}

The precise Hsu's method presented in this paper is an efficient method to determine the stability of the periodic solutions in which the matrix exponentials can be evaluated by the precise time integration algorithm. The advantage of this procedure is that it can minimize the round-off error for computing the transition matrix.

The considered numerical examples show that most of the periodic solution regions determined by the precise Hsu's method are in agreement with those determined by Hsu's method and the improved numerical integration scheme. However, the precise Hsu's method can correctly determine the stability of the critical stability-disputed regions so that it is superior to the other two approaches which give a wrong stability state in those regions.

\section{Acknowledgements}

Financial support from the National Natural Science Foundation of China (10672193) and the Research Grants Council of Hong Kong SAR (Project No. HKU7168/06E) is gratefully acknowledged.

\section{References}

1. C.S. Hsu, Impulsive parametric excitation: theory. ASME, Journal of Applied Mechanics 39, 1972, 551-558. 
2. C.S. Hsu, W.H. Cheng, Applications of the theory of impulsive parametric excitation and new treatments of general parametric excitation problems. ASME, Journal of Applied Mechanics 40, 1973, 78-86.

3. C.S. Hsu, On approximating a general linear periodic system. Journal of Mathematical Analysis Applications 45, 1974, 234-251.

4. P. Friedmann, C.E. Hammond, Efficient numerical treatment of periodic systems with application to stability problems. International Journal for Numerical Methods in Engineering 11, 1977, 1117-1136.

5. Y.K. Cheung, S.H. Chen and S.L. Lau, Application of the incremental harmonic balance method to cubic non-linearity systems. Journal of Sound and Vibration 140 (2), 1990, 273-286.

6. Z.Q. Cai, Y.X. Gu, W.X. Zhong, A new approach of computing Floquet transition matrix. Computers and Structures, 79, 2001, 631-635.

7. W.X. Zhong, On precise time-integration method for structural dynamics. Journal of Dalian University of Technology, 34(2), 1994, 131-135. (in Chinese)

8. W.X. Zhong, F.W. Williams. A precise time step integration method. Journal of Mechanical Engineering Science, 208, 1994, 427-430.

9. T.C. Fung, Z.L. Chen, Krylov precise time-step integration method. International Journal for Numerical Methods in Engineering, 68, 2006, 1115-1136.

10. S.Y. Zhang, Z.C. Deng, W.C. Li, A precise Runge-Kutta integration and its application for nonlinear dynamical systems. Applied Mathematics and Computation, 184, 2007, 496-502.

11. Y.A. Huang, Z.C. Deng, L.X. Yao, An improved symplectic precise integration method for analysis of the rotating rigid-flexible coupled system. Journal of Sound and Vibration, 299, 2007, 229-246.

12. M.F. Wang, F.T.K. Au, Assessment and improvement of precise time step integration method, Computers and Structures, 84, 2006, 779-786.

13. B. Tang, Combined dynamic stiffness matrix and precise time integration method for transient forced vibration response analysis of beams. Journal of Sound and Vibration, 309, 2008, 868-876.

14. B.S. Chen, L.Y. Tong, Y.X. Gu, Precise time integration for linear two-point boundary value problems. Applied Mathematics and Computation, 175, 2006, 182-211.

15. S.L. Lau, S.W. Yuen, Solution diagram of non-linear dynamic systems by the IHB method. Journal of Sound and Vibration, 167(2), 1993, 303-316.

16. S.L. Lau, Y.K. Cheung, S.Y. Wu, Incremental harmonic balance method with multiple time scales for aperiodic vibration of nonlinear systems. ASME Journal of Applied Mechanics, 50, 1983, 871-876.

17. S.H. Chen, Y.K. Cheung, H.X. Xing, Nonlinear vibration of plane structures by finite element and incremental harmonic balance method. Nonlinear Dynamics, 26(1), 2001, 87-104.

18. Y.Y. Lee, Structural-acoustic coupling effect on the nonlinear natural frequency of a rectangular box with one flexible plate. Applied Acoustics, 63, 2002, 1157-1175.

19. K.Y. Sze, S.H. Chen, J.L. Huang, The incremental harmonic balance method for nonlinear vibration of axially moving beams. Journal of Sound and Vibration, 281(3-5), 2005, 611-626,

20. C. Moler, C. Van Loan, Nineteen dubious ways to compute the exponential of a matrix, twentyfive years later. SIAM Review, 45(1), 2003, 1-46.

21. W.X. Zhong, On precise integration method. Journal of Computational and Applied Mathematics, 163, 2004, 59-78. 
Table I. The moduli of the eigenvalues by the three methods with $K$.

\begin{tabular}{|c|c|c|c|c|c|}
\hline $\begin{array}{c}\text { Number of integral } \\
\text { intervals } \\
K\end{array}$ & $\begin{array}{l}\text { Three points in } \\
\text { stability- } \\
\text { disputed regions }\end{array}$ & & $\begin{array}{l}\text { Improved numerical } \\
\text { integration } \\
\lambda_{1}=\lambda_{2}, \lambda_{3}=\lambda_{4}\end{array}$ & $\begin{array}{c}\text { Hsu's method } \\
\lambda_{1}=\lambda_{2}, \lambda_{3}=\lambda_{4}\end{array}$ & $\begin{array}{c}\text { The precise Hsu's } \\
\text { method }(N=20) \\
\lambda_{1}=\lambda_{2}, \lambda_{3}=\lambda_{4}\end{array}$ \\
\hline \multirow{3}{*}{400} & $\Omega=1.2070$ & $\begin{array}{l}\lambda_{1,2} \\
\lambda_{3,4}\end{array}$ & $\begin{array}{l}1.00000000381406 \\
0.99999993263521\end{array}$ & $\begin{array}{l}1.00000000451869 \\
0.99999993820133\end{array}$ & $\begin{array}{l}1.00000000000000 \\
1.00000000000000\end{array}$ \\
\hline & $\Omega=1.1819$ & $\begin{array}{l}\lambda_{1,2} \\
\lambda_{3,4}\end{array}$ & $\begin{array}{l}1.00000000091886 \\
0.99999997456974\end{array}$ & $\begin{array}{l}1.00000000103809 \\
0.99999997453354\end{array}$ & $\begin{array}{l}1.00000000000000 \\
1.00000000000000\end{array}$ \\
\hline & $\Omega=1.1165$ & $\begin{array}{l}\lambda_{1,2} \\
\lambda_{3,4}\end{array}$ & $\begin{array}{l}1.00000000251322 \\
0.99999995974264\end{array}$ & $\begin{array}{l}1.00000000219826 \\
0.99999996075951\end{array}$ & $\begin{array}{l}1.00000000000000 \\
1.00000000000000\end{array}$ \\
\hline \multirow{3}{*}{600} & $\Omega=1.2070$ & $\begin{array}{l}\lambda_{1,2} \\
\lambda_{3,4}\end{array}$ & $\begin{array}{l}1.00000000050245 \\
0.99999999112556\end{array}$ & $\begin{array}{l}1.00000000062210 \\
0.99999999183291\end{array}$ & $\begin{array}{l}1.00000000000000 \\
1.00000000000000\end{array}$ \\
\hline & $\Omega=1.1819$ & $\begin{array}{l}\lambda_{1,2} \\
\lambda_{3,4}\end{array}$ & $\begin{array}{l}1.00000000012102 \\
0.99999999665067\end{array}$ & $\begin{array}{l}1.00000000013667 \\
0.99999999664595\end{array}$ & $\begin{array}{l}1.00000000000000 \\
1.00000000000000\end{array}$ \\
\hline & $\Omega=1.1165$ & $\begin{array}{l}\lambda_{1,2} \\
\lambda_{3,4}\end{array}$ & $\begin{array}{l}1.00000000033106 \\
0.99999999469754\end{array}$ & $\begin{array}{l}1.00000000028096 \\
0.99999999484020\end{array}$ & $\begin{array}{l}1.00000000000000 \\
1.00000000000000\end{array}$ \\
\hline \multirow{3}{*}{800} & $\Omega=1.2070$ & $\begin{array}{l}\lambda_{1,2} \\
\lambda_{3,4}\end{array}$ & $\begin{array}{l}1.00000000011925 \\
0.99999999789377\end{array}$ & $\begin{array}{l}1.00000000014974 \\
0.99999999805963\end{array}$ & $\begin{array}{l}1.00000000000000 \\
1.00000000000000\end{array}$ \\
\hline & $\Omega=1.1819$ & $\begin{array}{l}\lambda_{1,2} \\
\lambda_{3,4}\end{array}$ & $\begin{array}{l}1.00000000002872 \\
0.99999999920515\end{array}$ & $\begin{array}{l}1.00000000003243 \\
0.99999999920403\end{array}$ & $\begin{array}{l}1.00000000000000 \\
1.00000000000000\end{array}$ \\
\hline & $\Omega=1.1165$ & $\begin{array}{l}\lambda_{1,2} \\
\lambda_{3,4}\end{array}$ & $\begin{array}{l}1.00000000007856 \\
0.99999999874161\end{array}$ & $\begin{array}{l}1.00000000006598 \\
0.99999999877618\end{array}$ & $\begin{array}{l}1.00000000000000 \\
1.00000000000000\end{array}$ \\
\hline \multirow{3}{*}{5000} & $\Omega=1.2070$ & $\begin{array}{l}\lambda_{1,2} \\
\lambda_{3,4}\end{array}$ & $\begin{array}{l}1.00000000000001 \\
0.99999999999978\end{array}$ & $\begin{array}{l}1.00000000000002 \\
0.99999999999979\end{array}$ & $\begin{array}{l}1.00000000000000 \\
1.00000000000000\end{array}$ \\
\hline & $\Omega=1.1819$ & $\begin{array}{l}\lambda_{1,2} \\
\lambda_{3,4}\end{array}$ & $\begin{array}{l}1.00000000000000 \\
0.99999999999992\end{array}$ & $\begin{array}{l}1.00000000000001 \\
0.99999999999992\end{array}$ & $\begin{array}{l}1.00000000000001 \\
1.00000000000000\end{array}$ \\
\hline & $\Omega=1.1165$ & $\begin{array}{l}\lambda_{1,2} \\
\lambda_{3,4}\end{array}$ & $\begin{array}{l}1.00000000000001 \\
0.99999999999987\end{array}$ & $\begin{array}{l}1.00000000000000 \\
0.99999999999987\end{array}$ & $\begin{array}{l}1.00000000000000 \\
1.00000000000000\end{array}$ \\
\hline
\end{tabular}



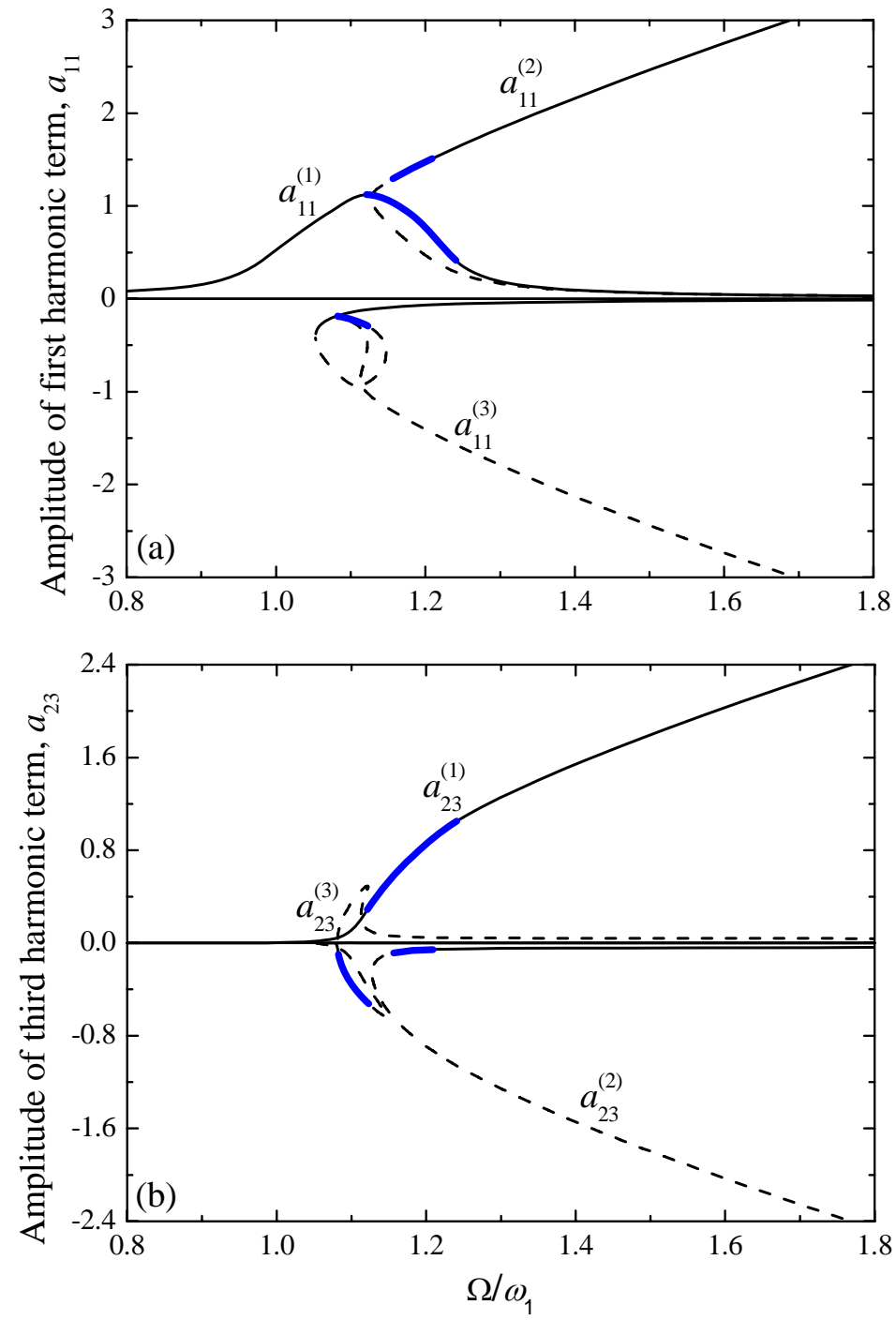

Figure 1. Forced frequency response with the IHB method with $f_{1}=0.03, \omega_{1} \approx 3 \omega_{2}, \Omega \approx \omega_{1}$.

(a) $\Omega-a_{11}$; (b) $\Omega-a_{23}$. 

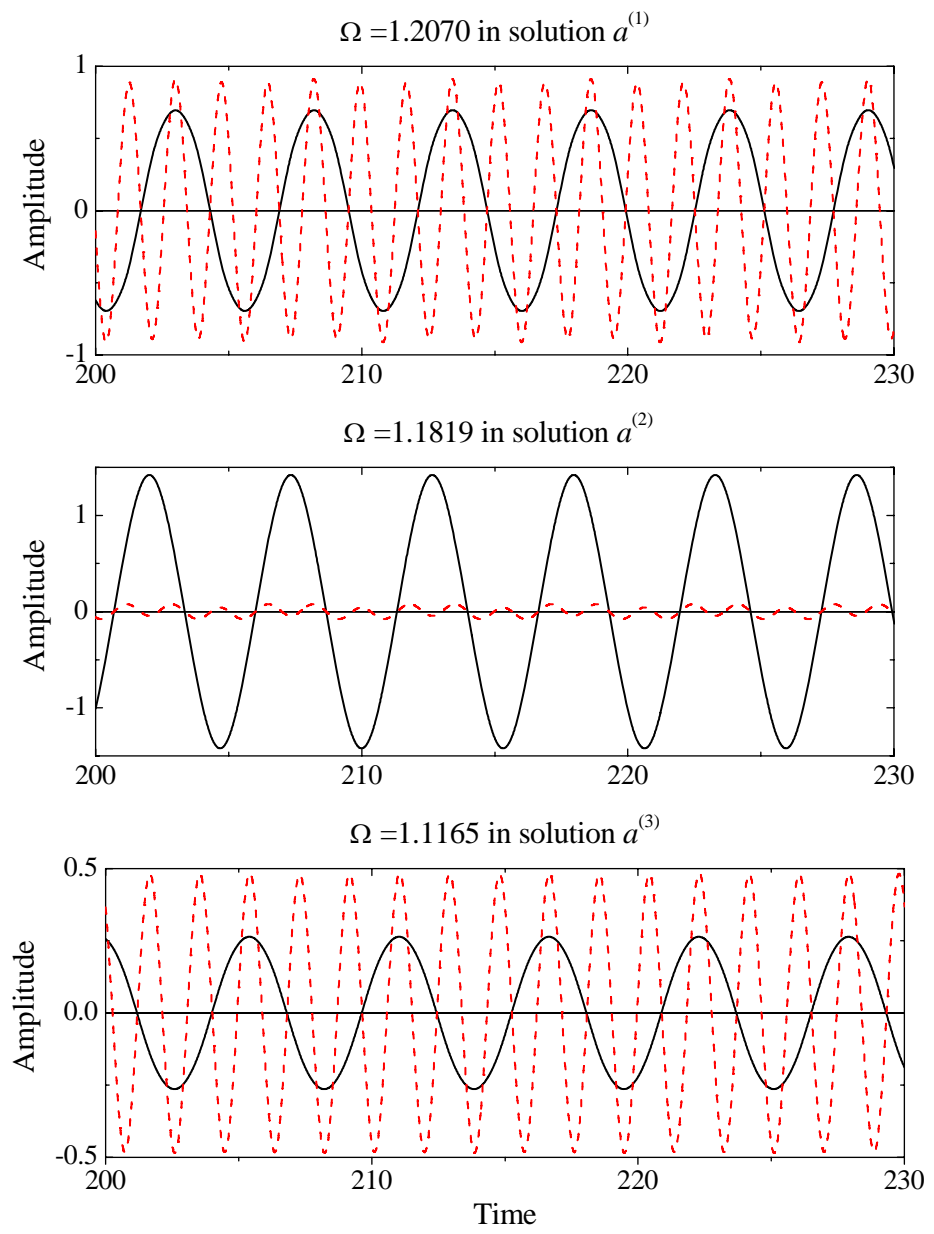

Figure 2. Existence of periodic motion of the clamped-hinged beam oscillations for three points in stability-disputed regions. $q_{1} ;-\cdots-\cdots q_{2}$. 


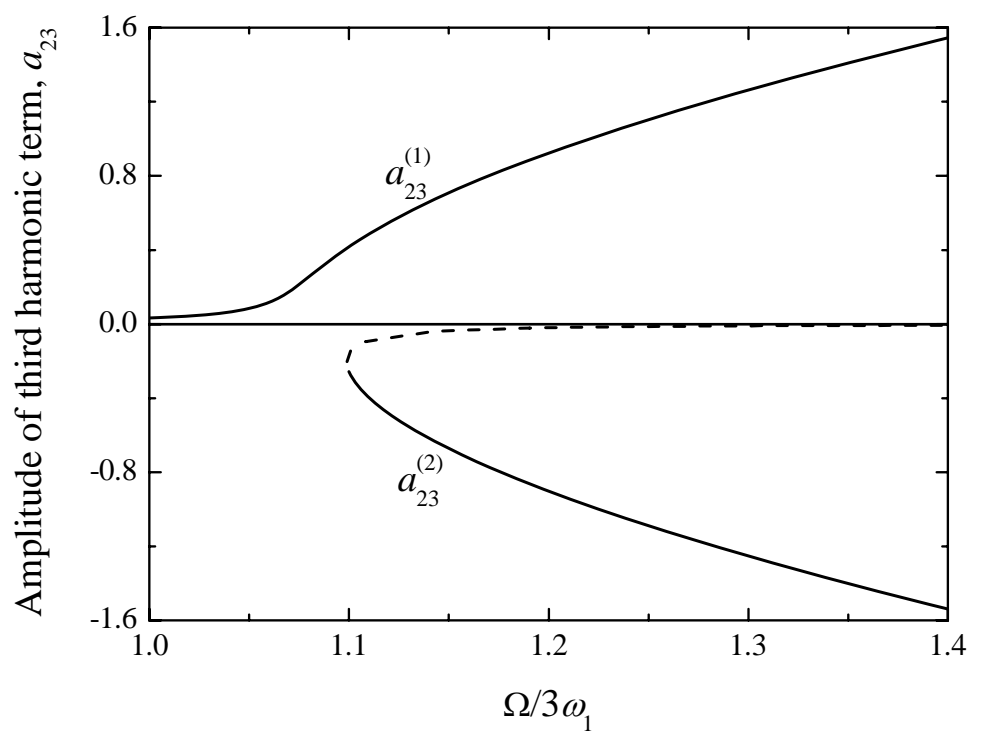

Figure 3. Fundamental resonance at $\Omega \approx \omega_{2}$ with the IHB method with $f_{2}=0.05, \omega_{2} \approx 3 \omega_{1}$. , stable; -----・, unstable. 

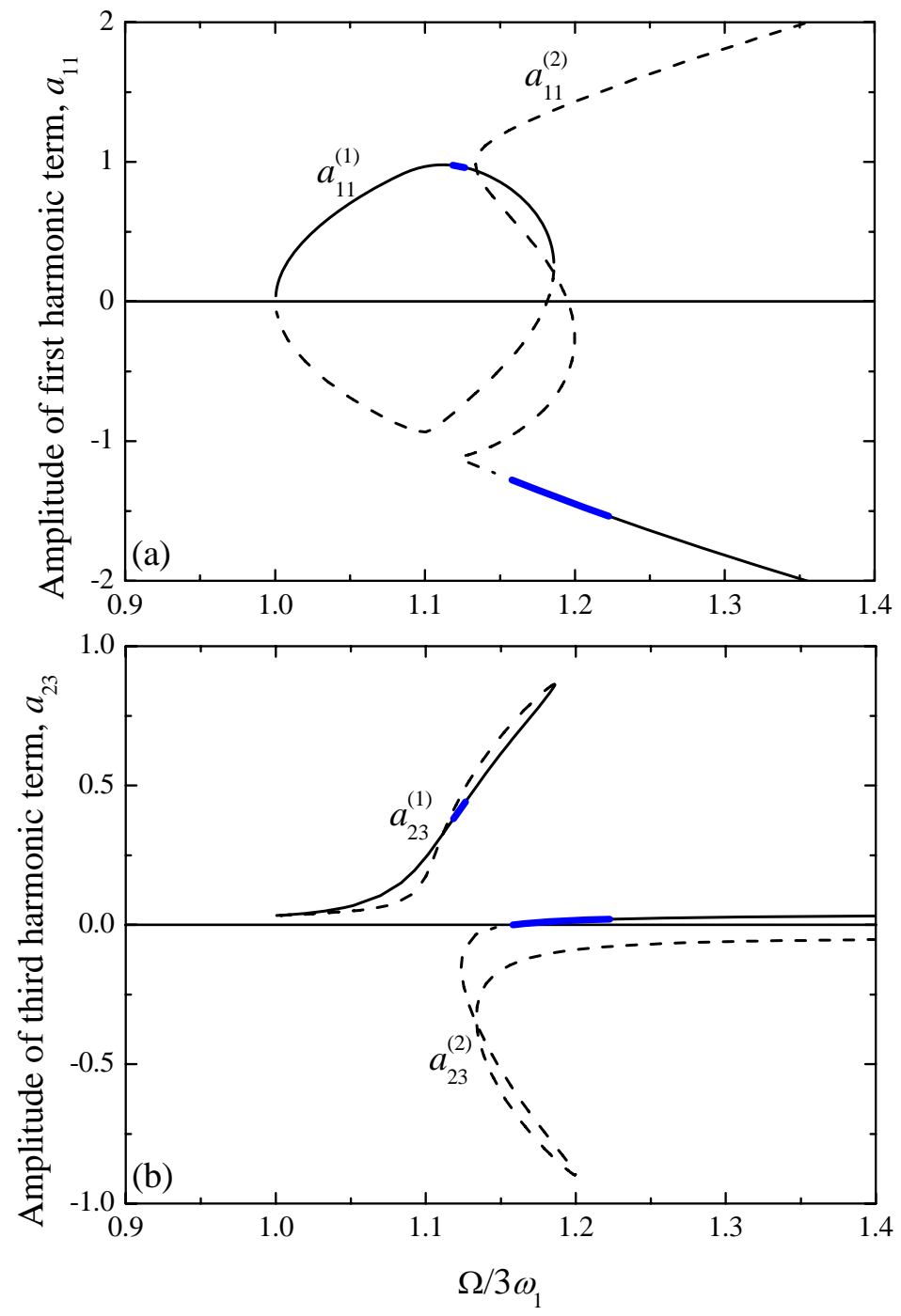

Figure 4. Subharmonic resonance at $\Omega \approx \omega_{2}$ with the IHB method with $f_{2}=0.05, \omega_{2} \approx 3 \omega_{1}$.

(a) $\Omega-a_{11}$; (b) $\Omega-a_{23}$. - , stable;---.-., unstable; - , stability disputed by the three methods. 

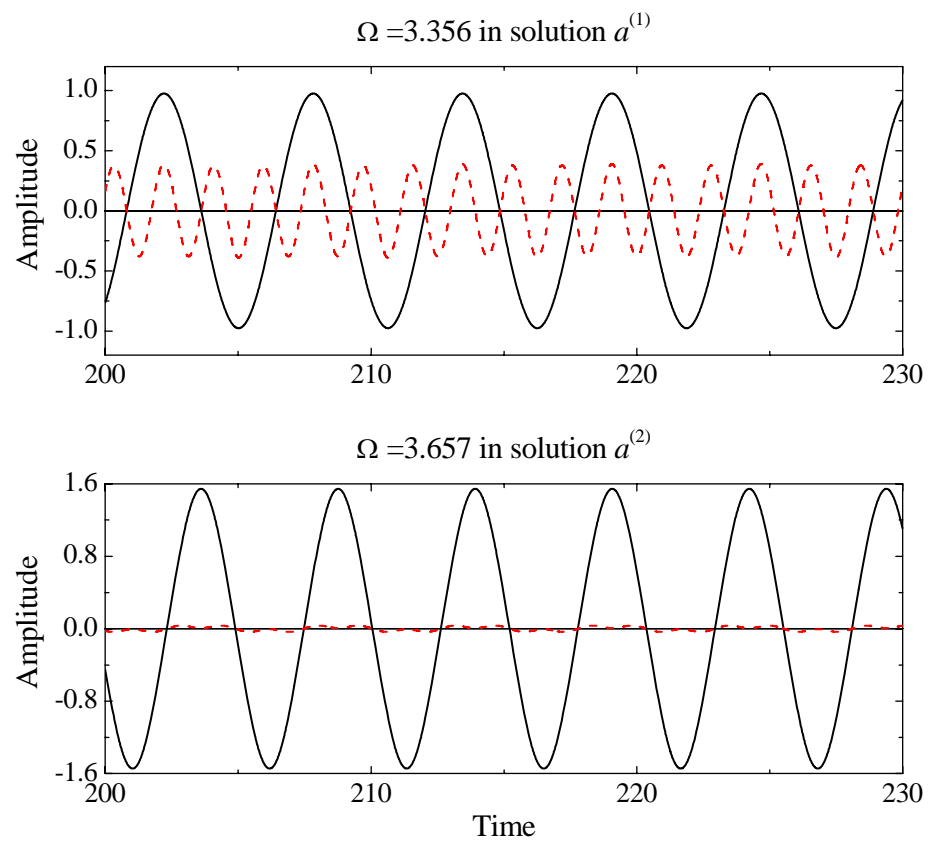

Figure 5. Existence of periodic motion of the clamped-hinged beam oscillations for two points in stability-disputed regions. $-q_{1} ;-\cdots q_{2}$. 